\title{
A CASE REPORT: PKP2 GENE C.1592T>G VARIATION IN HOMOZYGOUS FORM IDENTIFIED IN ARRHYTHMOGENIC RIGHT VENTRICULAR DYSPLASIA PATIENT
}

\author{
Luize Bidina ${ }^{1}$, Kaspars Kupics ${ }^{2}$, Emma Sokolova ${ }^{3}$, Mihails Pavlovics ${ }^{4}$, \\ Zane Dobele $^{5}$, Linda Piekuse ${ }^{6}$, Oskars Kalejs ${ }^{7}$
}

\begin{abstract}
Arrhythmogenic right ventricular dysplasia (ARVD) is an inherited cardiomyopathy. Early recognition and follow up of this disease can reduce sudden cardiac death burden. Arrhythmogenic right ventricular dysplasia is usually inherited as an autosomal dominant trait. We report a case of a young woman aged 26 years with a past history of chest pain and palpitations. During examination, abnormalities were found in results of an electrocardiogram and echocardiography. Genetic testing of the plakophilin 2 (PKP2) gene was done by direct sequencing and genetic variation "NG_009000.1: c.1592T>G" was found in a homozygote form. In family member screening in patients, parents' variation is found in a heterozygote form, where both are healthy. In all reports, "c. $1592 \mathrm{~T}>\mathrm{G}$ " is reported only in a heterozygous state, with no known pathogenicity. We consider that this is possibly a pathogenic mutation, inherited as an autosomal recessive trait.
\end{abstract}

UDC Classification: 612.1 DOI: http://dx.doi.org/10.12955/cbup.v4.823

Keywords: ARVD, PKP2, cardiovascular genetics.

\section{Introduction}

Arrhythmogenic right ventricular dysplasia (ARVD) is an inherited cardiomyopathy. Early recognition and follow up of this disease can reduce sudden cardiac death burden (Corrado, 2015). The development of ARVD is due to genetic mutations of desmosomal protein encoding genes (Corrado, 2015). A familial background has been demonstrated in over 50\% of ARVD cases. Arrhythmogenic right ventricular dysplasia is usually inherited as an autosomal dominant trait with incomplete penetrance and variable expression (Basso, 2009). It is unknown how many cases are caused by de novo mutations (McNally, 2014). Several studies have confirmed that Plakophilin 2 (PKP2) gene mutations in patients with ARVD are the most common ones, with a prevalence ranging from $11 \%$ to $51 \%$, mainly (up to $73 \%$ ) truncating mutations (Corrado, 2015; Basso, 2009; Dalal, 2006). Carriers with a missense variation are diagnosed with ARVD at an earlier age than carriers with stopgain mutations (LiMaura, 2013). The PKP2 mutations have been found in around $70 \%$ of cases with familial ARVD and no PKP2 mutations have been identifiable in cases with non-familial sporadic phenotype (Jain, 2008). Cases with PKP2 mutations are characterized with an earlier age of first clinical presentation and more frequent negative " $T$ " waves in right precordial leads (Alcalde, 2014). The aim of this case presentation is to describe a PKP2 genetic variation that has never been reported in a homozygote form and could possibly be pathogenic.

\section{Methods}

We examined a case of a young woman aged 26 years with a past history of chest pain and palpitations. During an examination, the woman had presented with abnormalities identified by electrocardiogram and echocardiography.

The diagnoses of ARVD were made according to Revised Task Force Criteria (Frank, 2010). Several investigations were performed by electrocardiogram (ECG), transthoracic echocardiography (TTE), 24-hour Holter monitoring, cardiac magnetic resonance, and exercise ECG as suggested in guidelines (Corrado, 2015). The DNA from the patient, her family members and control individuals were isolated

\footnotetext{
${ }^{1}$ Luize Bidina, Riga Stradins university, Latvia, luizebidina@gmail.com

${ }^{2}$ Kaspars Kupics, Pauls Stradins Clinical University Hospital, Latvia, kaspars.kupics@gmail.com

${ }^{3}$ Emma Sokolova, Pauls Stradins Clinical University Hospital, Latvia, emma.sokolova@inbox.lv

${ }^{4}$ Mihails Pavlovics, Pauls Stradins Clinical University Hospital, Latvia, mihails.pavlovics@ gmail.com

${ }^{5}$ Zane Dobele, Scientific labaratory of molecular genetics, Riga Stradins university, Latvia, zane.dobele @ gmail.com

${ }^{6}$ Linda Piekuse, Head of Scientific labaratory of molecular genetics, Riga Stradins university, Latvia,

linda.piekuse@gmail.com

${ }^{7}$ Oskars Kalejs, Department of Arrhythmology, Pauls Stradins Clinical University Hospital, Latvia, okalejs@ gmail.com
} 
by standard phenol chloroform method (Sambrook, 2006) from venous blood with ethylenediaminetetraacetic acid (EDTA) anticoagulant. Direct sequencing of the PKP2 gene (Gene Bank Accession no: NC 000012) was done. Primer sequences were adapted from a publication (Gerull, 2004). Sequences were verified in the Basic Local Alignment Search Tool (BLAST) database and compared to the PKP2 gene reference sequences (NM_004572.3 and NG_009000.1). The discovered genetic variations were verified in the ARVD database (van der Zwaag et al., 2009) to study their possible connection with ARVD. Genetic variations in minor allele frequency (MAF) were compared with European population MAF, using data from the "1000 Genome" project browser (Auton A et al., 2015). The 7th exon was analyzed in 50 unaffected Latvian individuals.

\section{Results}

The ECG showed T-wave inversion in right precordial leads. In the TTE, a right ventricle (RV) aneurysm was found and a parasternal long-axis (PLAX) right ventricular outflow (RVOT) greater than $29 \mathrm{~mm}$, parasternal short-axis (PSAX) RVOT greater than $36 \mathrm{~mm}$, and a ratio of PSAX to body surface area (BSA) greater than $21 \mathrm{~mm}$. These results indicated major criteria in ECG and TTE. In the cardiac magnetic resonance, RV ejection fraction (EF) was $61.44 \%$, the ratio of right ventricular enddiastolic volume (RVEDV) to BSA was $74.17 \mathrm{~mL} / \mathrm{m}^{2}$, but there were no intra-myocardial morphological abnormalities, which include intra-myocardial fatty infiltration, focal wall thinning, wall hypertrophy, and trabecular hypertrophy (Tandri, 2004). In the 24-hour Holter monitoring and exercise with ECG, observations were normal. After the first annual visit, there was no noteworthy changes in ECG, TTE, and Holter monitoring, and the patient presented with no complaints.

After genetic analyses two non-pathogenic genetic variations were found: "NM_004572.3: c.2145+45G $>$ A" (rs10772008) and "NM_004572.3:c.2578-69G $>A$ " (rs7956824). One novel, unregistered, possibly non-pathogenic genetic variation "c.2489+131G $>A$ " was discovered. One genetic variation was found in a homozygote variation, "c.1592T $>\mathrm{G}$ " (Figure 1).

Figure 1: Genetic variation c. $1592 \mathrm{~T}>\mathrm{G}$

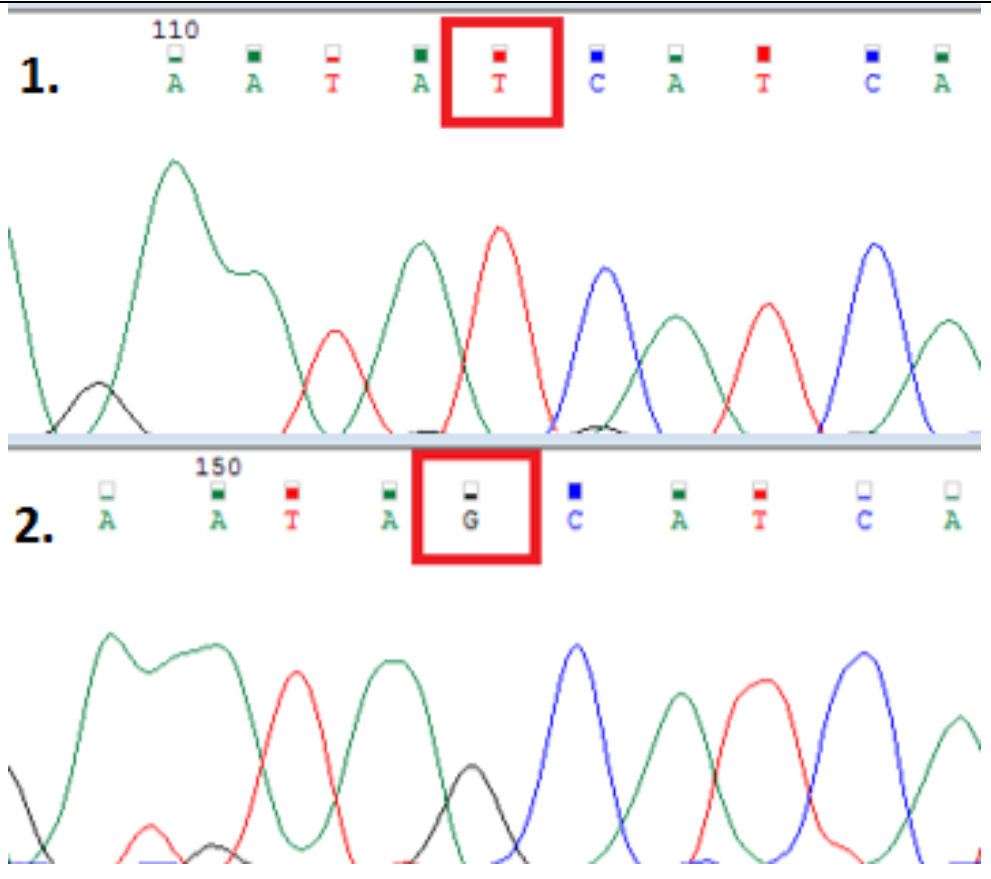

1. Showed reference sequence - without $c .1592 \mathrm{~T}>\mathrm{G}$ variation

2. Patients sequence $-\mathrm{c} .1592 \mathrm{~T}>\mathrm{G}$ in homozygous form.

Source: Author

It was a missense mutation in 7th exon, and in the ARVD mutation database it is described as not a known pathogenicity. However, in all reports, it was reported only in the heterozygous state, but in our patient it was in the homozygous state, which could possibly be pathogenic. The genetic variation 
minor allele frequency (MAF) in Latvian ARVD registry patients was 0.05 . In a healthy Latvian control group $\mathrm{MAF}_{\mathrm{LV}}$ is zero. A comparison of the MAF of our registry patients to that of the European population, reveals an $\mathrm{MAF}_{\mathrm{EU}}$ of 0.006 , p-value 0.0005, Odds ratio (OR) 12.5 (CI 95\% 351.8). In the index, the patients' parents were from an unrelated marriage and "c.1592T>G" was found in the heterozygote form. Both, mother and father, were healthy, and had no complaints of palpitations, chest pain, or syncope. Any other first degree relative was not affected. There was no family history of ARVD in a first-degree relative or premature sudden death $(<35$ years of age $)$ due to a suspected arrhythmogenic right ventricular cardiomyopathy or dysplasia (ARVC/D).

\section{Conclusion}

For the first time, genetic variation, c. $1592 \mathrm{~T}>\mathrm{G}$, has been discovered and reported in the homozygote form. It was statistically significant that $c .1592 \mathrm{~T}>\mathrm{G}$ was more common in ARVD patients than in healthy controls. We consider that the genetic variation c. $1592 \mathrm{~T}>\mathrm{G}$ is inherited as an autosomal recessive trait. This genetic variation is possibly pathogenic and has a high probability of being approved for genetic ARVD diagnosis.

\section{References}

Auton A, (2015) A global reference for human genetic variation, The 1000 Genomes Project Consortium, Nature 526, 6874,doi:10.1038/nature15393

Alcalde, M. (2014). Stop-Gain Mutations in PKP2 Are Associated with a Later Age of Onset of Arrhythmogenic Right Ventricular Cardiomyopathy. PLoS One, 26;9(6), 560.

Basso, C. (2009). Arrhythmogenic right ventricular cardiomyopathy. Lancet, 373, 1289-1300.

Corrado, D. (2015). Treatment of Arrhythmogenic Right Ventricular Cardiomyopathy/Dysplasia. Circulation, 132(5), 441453.

Dalal, D. (2006). Clinical features of arrhythmogenic right ventricular dysplasia/cardiomyopathy associated with mutations in plakophilin-2. Circulation, 113, 1641-1649.

Frank, I. (2010). Diagnosis of arrhytmogenic right ventricular cardiomyopathy/dysplasia. Proposed Modification of the Task Force Criteria. Eur Heart J., 31(7):806-814

Gerull, B. (2004). Mutations in the desmosomal protein plakophilin-2 are common in arrhythmogenic right ventricular cardiomyopathy. Nat Genet, 36(11), 1162-1164

Jain, A. (2008). Role of cardiovascular magnetic resonance imaging in arrhythmogenic right ventricular dysplasia. $J$ Cardiovasc Magn Reson, 10(1), 32.

LiMaura, I. E. (2013). Identification of a PKP2 gene deletion in a family with arrhythmogenic right ventricular cardiomyopathy. Eur J Hum Genet, 21(11), 1226-1231.

McNally, E. (9. January 2014). Arrhythmogenic Right Ventricular Dysplasia/Cardiomyopathy. Načteno z PubMed: http://www.ncbi.nlm.nih.gov/books/NBK1131/

Sambrook, J. (2006). Purification of nucleic acids by extraction with phenol: chloroform. CSH Protoc, 10, 1101.

Tandri, H. (2004). Magnetic resonance and computed tomography imaging of arrhythmogenic right ventricular dysplasia. $J$ Magn Reson Imaging, 19(6), 848-858.

van der Zwaag, P. A (2009). A genetic variants database for arrhythmogenic right ventricular dysplasia/cardiomyopathy. Hum Mutat. 30(9), 1278-83. 